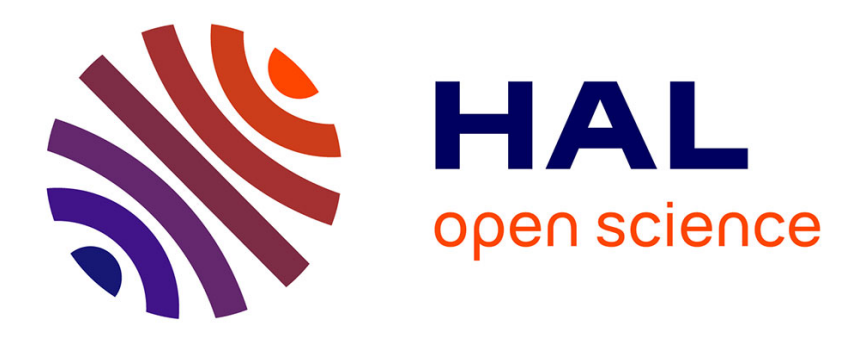

\title{
Plasma de césium ionisé par contact dans une machine à confinement magnétique: Pascal
}

\author{
H. Doucet, H. Lamain
}

\section{To cite this version:}

H. Doucet, H. Lamain. Plasma de césium ionisé par contact dans une machine à confinement magnétique: Pascal. Revue de Physique Appliquée, 1967, 2 (3), pp.153-157. 10.1051/rphysap:0196700203015300 . jpa-00242782

HAL Id: jpa-00242782

https://hal.science/jpa-00242782

Submitted on 1 Jan 1967

HAL is a multi-disciplinary open access archive for the deposit and dissemination of scientific research documents, whether they are published or not. The documents may come from teaching and research institutions in France or abroad, or from public or private research centers.
L'archive ouverte pluridisciplinaire HAL, est destinée au dépôt et à la diffusion de documents scientifiques de niveau recherche, publiés ou non, émanant des établissements d'enseignement et de recherche français ou étrangers, des laboratoires publics ou privés. 


\title{
REVUE DE PHYSIQUE APPLIQUÉE
}

\author{
Supplément au "Journal de Physique »
}

\section{PLASMA DE GÉSIUM IONISÉ PAR GONTAGT DANS UNE MAGHINE A GONFINEMENT MAGNÉTIQUE : PASGAL (1)}

\author{
Par H. DOUGET et H. LAMAIN, \\ Laboratoire de Physique des Milieux Ionisés, École Polytechnique, Paris \\ (Équipe de recherche associée au G.N.R.S.).
}

\begin{abstract}
Résumé. - On décrit une machine produisant un plasma de césium ionisé par contact confiné dans un champ magnétique. Entièrement démontable, fonctionnant en pression de vapeur saturante, la machine Pascal est construite pour l'étude des ondes ioniques et des instabilités électrostatiques qui y sont associées.
\end{abstract}

\begin{abstract}
A cesium plasma device is described in which surface ionization and magnetic confinement are used. The device called Pascal is entirely demountable, working in a vaporpressure mode and has been built for the sudy of ion-wave and associated electrostatic instabilities studies.
\end{abstract}

1. Introduction. - Dans cet article, nous présentons une machine appelée Pascal, produisant un plasma de césium ionisé par contact et confiné dans un champ magnétique.

Après les travaux très complets de Langmuir et Kingdon [1] de 1923 à 1937, puis les travaux sur la recombinaison de Mohler [2], on disposait de toutes les connaissances technologiques nécessaires à la réalisation d'un plasma par ionisation de contact dès 1937 . Ge n'est qu'en 1955 que Dreicer [3] montra l'intérêt d'un plasma de césium en équilibre thermodynamique avec les parois. Les difficultés de réalisation d'une telle enceinte portée à quelque $2500 \mathrm{~K}$ ou plus firent d'abord choisir d'autres types de production. En 1958, Knechtli et Knauer [4] d'une part, Hernquist [5] d'autre part, réalisèrent des plasmas de césium en tubes scellés en présence de champ magnétique. Ces travaux permirent la réalisation des grosses machines au césium puis avec d'autres alcalins et les premiers alcalino-terreux, d'abord à Princeton avec les «machines $Q$ » [6], puis un peu partout dans le monde. Dans les machines du type $Q$, l'injection de césium neutre est une des plus grosses difficultés. En effet, il est difficile de produire un faisceau de neutres très homogène et encore plus d'obtenir deux faisceaux de neutres identiques; si bien que, avec ce type de machine, il existe toujours une vitesse moyenne du plasma qui intervient directement dans les mesures de vitesse de phase des ondes ioniques et du taux d'amortissement [7].

( $\left.{ }^{1}\right)$ La construction du dispositif Pascal a été réalisée avec l'appui financier de la D.G.R.S.T. et de la D.R.M.E.
D'autre part, en injection directe, il peut apparaître une instabilité résistive qui ne semble pas exister dans les machines fonctionnant en pression de vapeur saturante [8].

Nous avons donc choisi de construire une machine produisant un plasma de caractéristiques analogues à celles des plasmas des machines Q1 et Q3 de Princeton, mais fonctionnant en pression de vapeur saturante.

De plus, de manière à pouvoir modifier les dispositions des électrodes internes, on a prévu un ensemble entièrement démontable.

2. Description du dispositif. - Le dispositif Pascal comprend une enceinte à vide étuvable fermée par une vanne à joint métallique et comportant un appendice qui renferme le césium. Le plasma confiné est limité longitudinalement par deux cathodes chauffées par bombardement électronique et radialement par le champ magnétique.

L'ensemble du dispositif est conforme au schéma présenté sur la figure 1.

A. Émetteurs. - Les émetteurs sont formés par deux cathodes en tantale chauffées par bombardement électronique. Ces cathodes sont réalisées par un disque de diamètre $28 \mathrm{~mm}$ et d'épaisseur $5 \mathrm{~mm}$, monté sur une jupe de longueur $10 \mathrm{~cm}$ et d'épaisseur $0,2 \mathrm{~mm}$. Elles sont identiques à la cathode [9] utilisée sur le dispositif OPS décrit par ailleurs [10]. Afin de pouvoir fonctionner en vapeur de césium, à des potentiels variables, les émetteurs sont montés sur des bagues en céramique et des supports en acier inoxydable. 

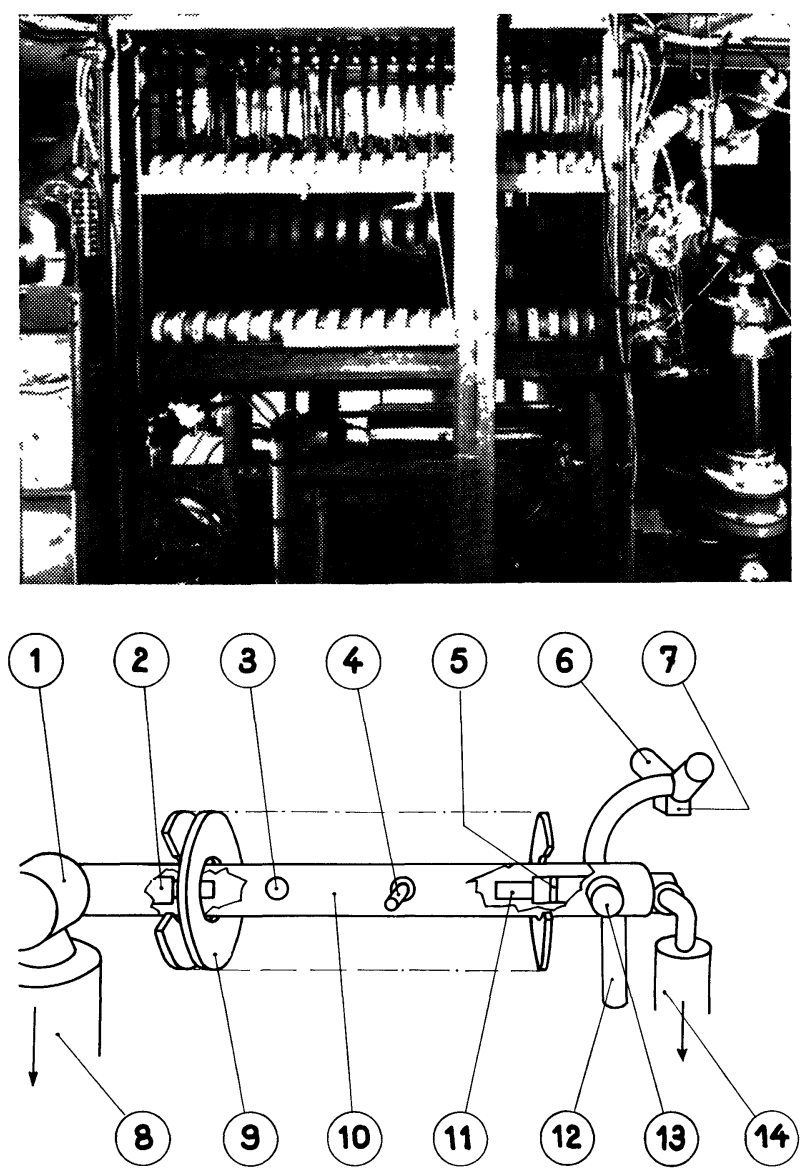

Réalisées par la G.E.P.E.C. à Gorbeville, ces cathodes ont fonctionné durant 500 heures environ à $2000^{\circ} \mathrm{C}$ sans dommage apparent. La figure 2 donne une vue d'ensemble de l'une des cathodes et des détails de montage.
FIG. 1. - Pascal : schéma d'ensemble du dispositif.

1. Vanne à joint métallique étuvable Varian.

2. Support de cathode.

3. Passage démontable.

4. Support de sonde électrostatique mobile.

5 Bague d'alumine pour l'isolement de la cathode.

6. Spectromètre de masse analyseur de gaz Veeco.

7. Aimant permanent.

8. Pompage principal à diffusion d'huile.

9. Bobines créant le champ magnétique.

10. Enceinte en acier inoxydable étuvable.

11. Cathode en tantale chauffée par bombardement électronique.

12. Réserve de césium à tempétature contrôlée. 13. Jauge à ionisation type Bayard-Alpert.

14. Pompage arrière des cathodes à diffusion d'huile.
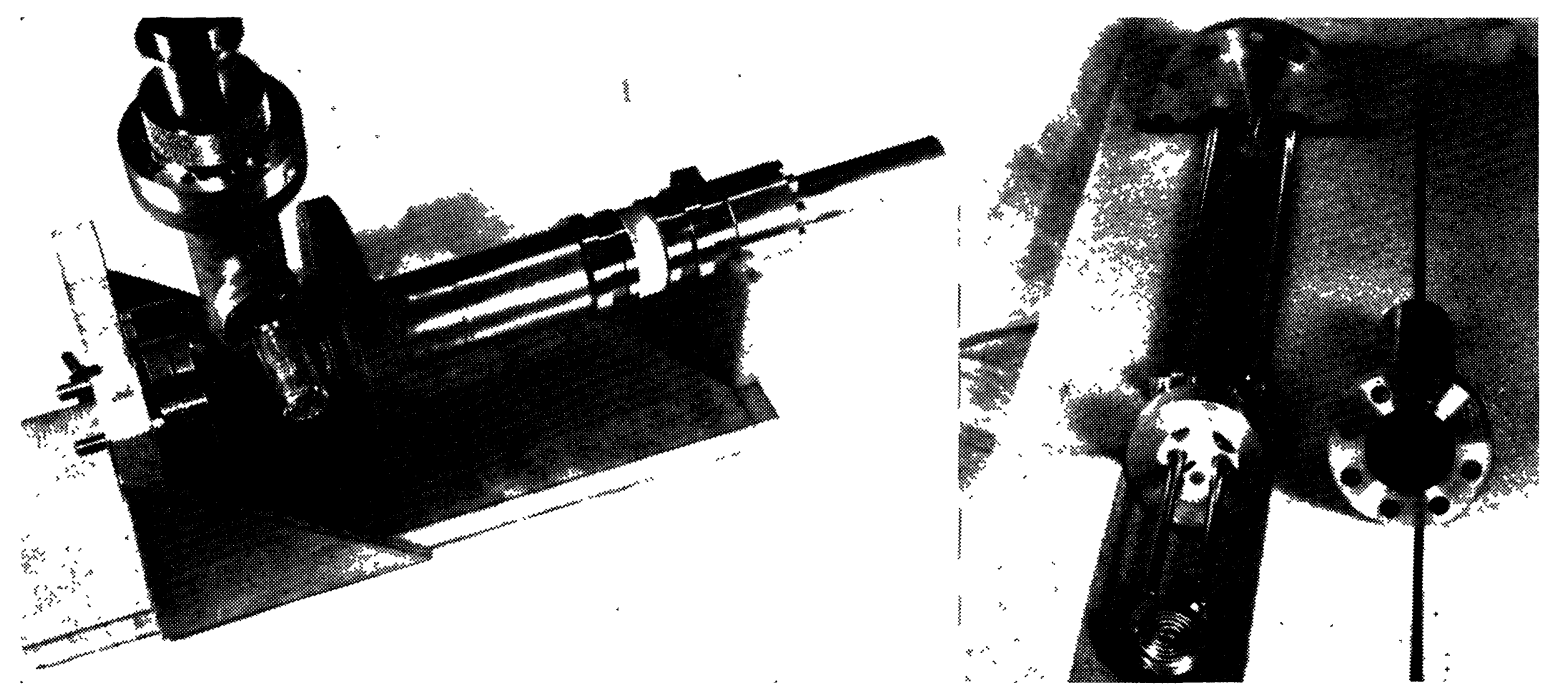

Fig. 2. - Cathode chauffée par bombardement électronique. Détails des filaments. 
une vanne Varian étuvable de diamètre $10 \mathrm{~cm}$. Chaque élément est chauffé par un circuit électrique séparé et maintenu à la température désirée par une régulation. L'évolution des températures est suivie par un enregistreur à 12 voies.

G. Pompage. - Le pompage est assuré par un ensemble de deux pompes à diffusion d'huile, en série, surmonté d'un baffle à eau. Le vide limite obtenu avec l'huile convalex 10 est de $3 \cdot 10^{-9}$ torr au bout de l'enceinte après un bon dégazage. Vanne fermée, la pression remonte jusqu'à $10^{-6}$ torr au bout d'une heure environ. Les expériences sont faites pendant la remontée de la pression. Quand la pression des gaz résiduels n'est plus très faible devant la pression de vapeur saturante du césium, il suffit de rouvrir la vanne pendant quelques minutes pour retrouver un bon vide.

D. Injegtion De Gésium. - Deux dispositifs ont été prévus :

- Pression de vapeur saturante. - Un appendice en acier inoxydable dont la température est contrôlable par un four à thermostat contient une ampoule de césium scellée et un morceau de nickel. Lorsqu'on désire introduire le césium, on brise l'ampoule à l'aide d'un morceau de nickel qui est manœuvrable de l'extérieur de l'enceinte par un aimant. Une petite vanne Varian étuvable de diamètre $3,5 \mathrm{~cm}$ permet d'isoler l'appendice.

- Injection directe sur un émetteur. - Le césium est alors transporté depuis une enceinte à température contrôlée, jusqu'à un diffuseur placé autour des cathodes, par un tube chauffé.

Pour obtenir un plasma assez homogène, nous utilisons un diffuseur circulaire entourant une cathode, percé de 8 trous, projetant le césium sur l'émetteur.

La répartition de densité obtenue est indiquée sur la figure 3 . Ce système d'injection a été mis au point et utilisé sous vide classique dans le dispositif OPS décrit par ailleurs [10].

E. Champ magnétique. - Un champ magnétique variable de 0 à 5 kilogauss, uniforme à $3 \%$ près dans tout le volume du plasma, est parallèle à l'axe des émetteurs.

En conclusion, le dispositif Pascal permet de produire, dans des conditions bien définies, un plasma de césium ionisé par contact. Il possède, par rapport aux machines actuellement existantes, les deux particularités suivantes :

- fonctionnement possible en pression de vapeur saturante pour les basses densités $\left(n_{0} \leqslant 10^{11} \mathrm{p} / \mathrm{cm}^{3}\right)$, ce qui produit un plasma très homogène ;

- pompage séparé des cathodes, permettant d'utiliser un vide classique pour le bombardement électronique. Cette disposition interdit aux électrons rapides qui pourraient contourner la cathode de pénétrer le plasma, comme Rogers a pu l'observer [12].

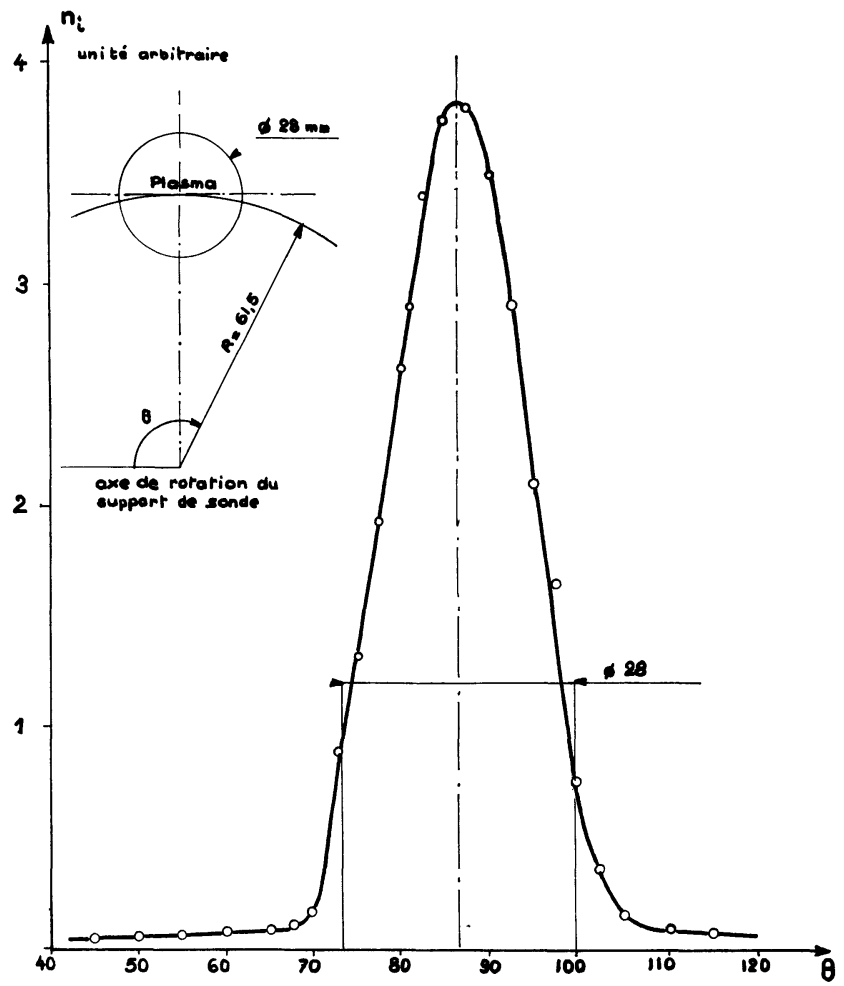

FIG. 3. - Exemple de répartition radiale de densité obtenue en injection directe.

3. Diagnostic. - Le diagnostic du plasma est réalisé à l'aide de sondes de Langmuir du type classique, formées d'un fil de tungstène très fin (1/100 à $1 / 10$ de mm) placé dans un tube d'alumine pure.

Les caractéristiques de sondes relevées sont assez proches des caractéristiques classiques et comportent une partie exponentielle qui donne une valeur de la température électronique égale à la température des cathodes quand le plasma est calme.

Les densités ioniques mesurées par ces caractéristiques donnent des densités qui restent inférieures à $10^{12} \mathrm{p} / \mathrm{cm}^{3}$.

Une méthode d'interférométrie classique microondes en $8 \mathrm{~mm}$ a été prévue pour mesurer les densités électroniques.

Des hublots en verre comportant des liaisons verrecuivre sont utilisés pour ces mesures et ont fonctionné plusieurs heures à $150^{\circ} \mathrm{C}$ en présence de césium sans dommage apparent.

La pression de césium neutre est connue par la tension de vapeur correspondant à la température de l'appendice césium, mais la pression de césium est lente à s'établir, probablement par suite des adsorptions et désorptions des parois. La jauge BayardAlpert, utilisée pour donner la pression des gaz résiduels, est fortement perturbée en présence de césium et ne fournit aucune indication valable. Les pressions partielles des gaz de désorption et de césium sont 
contrôlées à l'aide d'un analyseur de gaz Veeco du type spectromètre de masse à $60^{\circ}$.

La pression de vapeur de césium est également connue par la mesure du courant maximum qui circule entre les deux émetteurs quand on applique une différence de potentiel de quelques volts entre deux émetteurs. En effet, la mesure du courant de saturation constitue alors une mesure absolue de la pression de vapeur alcaline. Les variations de courant en fonction du potentiel sont indiquées en figure 4 .

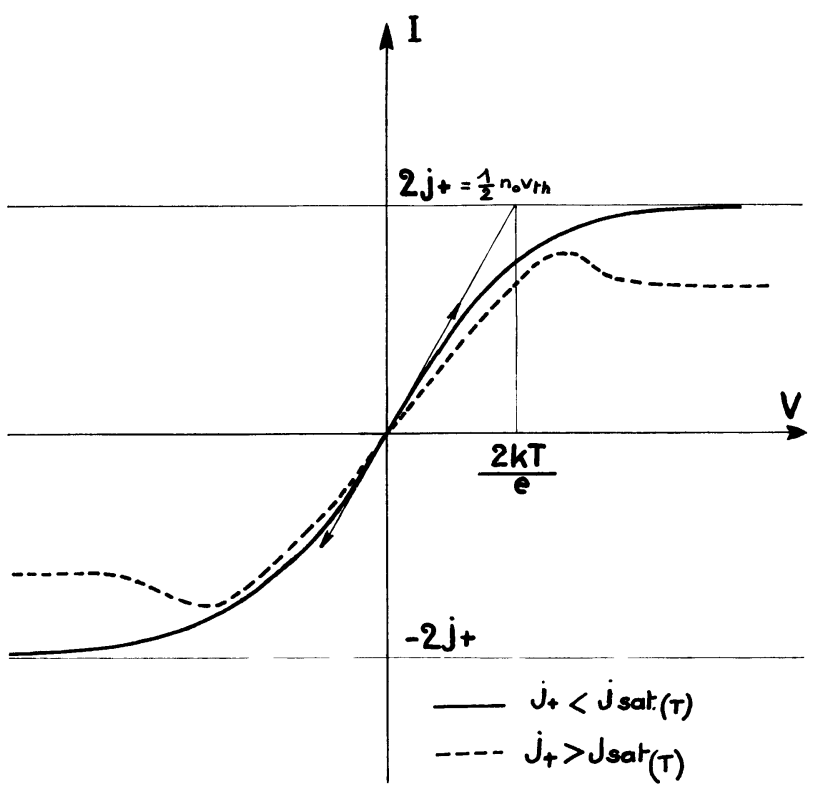

FIG. 4. - Variations du courant avec la tension appliquée entre les deux émetteurs.

Les caractéristiques principales du plasma obtenu sont les suivantes :

- nature : césium;

- injection : pression de vapeur saturante;

- longueur max. du cylindre de plasma : $80 \mathrm{~cm}$;

- diamètre max. du cylindre de plasma : $2,8 \mathrm{~cm}$;

- champ magnétique : minimum 0 , maximum $5 \mathrm{kG}$;

- homogénéité du champ magnétique : $3 \%$;

- température électronique et ionique max. : $2500^{\circ} \mathrm{K}$;

- taux d'ionisation : minimum $0 \%$, maximum $\sim 99 \%$;

- densité électronique : minimum $\sim 10^{6} \mathrm{p} / \mathrm{cm}^{3}$, maximum $\sim 10^{12} \mathrm{p} / \mathrm{cm}^{3}$

- pression des gaz résiduels min. $\sim 3 \times 10^{-9}$ torr.

4. Premières expériences de propagation. - En appliquant une tension pulsée sur une grille plongée dans le plasma et polarisée négativement, on produit une perturbation de densité ionique qui se propage dans le plasma. Cette perturbation est détectée sur une seconde grille analogue à la première. La figure 5

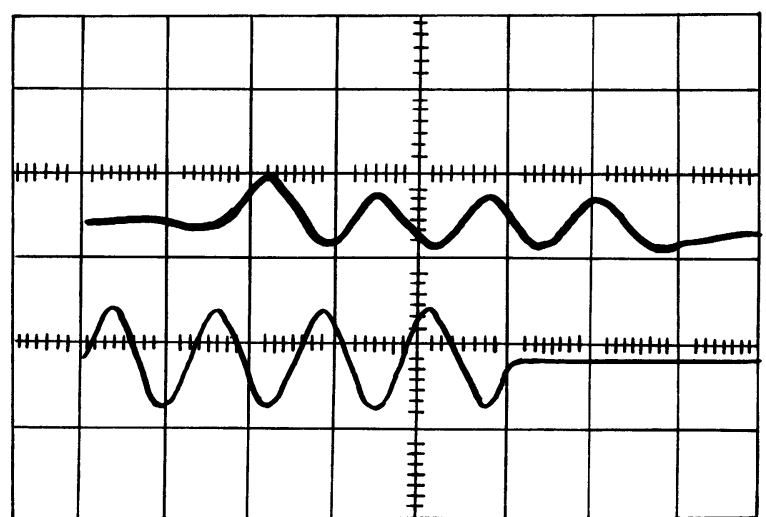

FIG. 5. - Exemple de signaux d'excitation et de détection :

- Trace inférieure : tension d'excitation : $5 \mathrm{~V} /$ carreau. - Trace supérieure : tension détectée : $10 \mathrm{mV} /$ carreau. Balayage horizontal : $100 \mu \mathrm{s} /$ carreau.

donne un exemple des signaux appliqués et obtenus. On constate que :

1. Le signal détecté est semblable au signal d'excitation, ce qui semble montrer que la propagation se fait sans dispersion, les vitesses de phase et de groupe étant égales.

2. Les vitesses de phase mesurées dans les deux sens possibles de propagation en inversant le rôle des deux grilles sont égales lorsque le courant est annulé en ajustant les températures des cathodes. Ceci montre qu'il n'y a pas de vitesse moyenne du plasma.

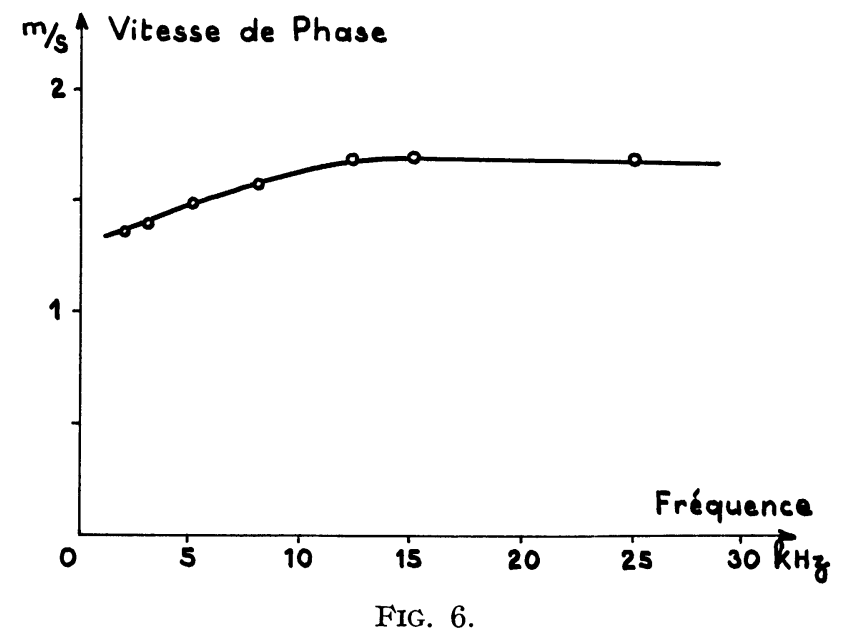

Variations de la vitesse de phase avec la fréquence.

3. Les vitesses de phase varient très peu avec la fréquence, comme le montre la figure 6 . En particulier, il n'y a pas de dispersion appréciable au voisinage de la fréquence cyclotronique des ions. La propagation semble donc être plane. 
4. La vitesse de phase mesurée semble indépendante de la densité du plasma et du champ magnétique.

5. La valeur de cette vitesse de phase est en bon accord avec la valeur calculée par d'Angelo, Wong et Motley [7].

En conclusion : la propagation d'une onde ionique plane a été réalisée dans notre machine de façon satisfaisante. Les premières mesures sur les vitésses de phase montrent que l'effet des vitesses moyennes du plasma y est négligeable quand on annule le courant. Cette situation expérimentale doit nous permettre d'étudier de façon approfondie l'amortissement des ondes et leur propagation en présence de courant.

Manuscrit reçu le 15 avril 1967.

\section{BIBLIOGRAPHIE}

[1] Langmuir (I.), Kingdon, Phys. Rev., 1923, 21, 380 ; Science, 1923, 57, 58.

Langmutr (I.), KIngdon, Proc. Royal Soc., 1925, A, CVII, 61 .

LANGMUIR (I.) et "Collected Works ", 3, 273, Pergamon Press, 1961.

[2] Mohler (F. L.), J. Res. Nat. Bur. Standards, 1937, 19, 447, 559 .

MOHLER (F. L.), BOECKNER (C), J. Res. Nat. Bur. Standards, 1929, 2, 489.

[3] Dreicer (H.), Plasma in equilibrium, Proc. of the Conf. on Contr. Thermo. Reactions, Princeton, 1955, 507-511 (TID-7503).

[4] KNechtil (R. C.), KNauer (W.), Bull. Amer. Phys. Soc , 1958, 3, 81.

KNECHTLI (R. C.), WADA (J. Y.), Bull. Amer. Phys. Soc., 1960, 5, 366.

KNECHTLI (R. C.), WADA (J. Y.), 5 e C.I.P.I.G., Munich, 1961, I, 786.

WAdA (J. Y), KNeChTLI (R. C.), Proc. of the I.R.E., 1961, 49, 1926.
[5] HERNQUiST (K. G.), KANELSHY (M.), NORMAN (F.H.), R.C.A. Rev., 1958, 19, 244.

[6] Rynn (N.), d'ANgero (N.), Rev. Sci. Instr., 1960, 31, 1326.

[7] WONG (A. Y.), MOTLLEY (R. W.), d'ANGEIO (N.), Phys. Rev., 1964, 133, 436.

[8] Douce'T (H.), Jones (W. D.), C. R. Acad. Sci. Paris, 1965, 260, 3577 ; C. R. Acad. Sci. Paris, 1965, 261, 2862.

Doucet (H.), J. Physique, 1965, 26, 514, 7e Conf. Inter. sur les Phénomènes d'Ionisation dans les Gaz (C.I.P.I.G.), Belgrade, 1965.

[9] Douce'T (H.), Lamain (H.), Laboratoire de Physique des Milieux Ionisés, École Polytechnique, Rapp. int. no 277, 1965.

[10] A paraître au Journal de Physique.

[11] Galiais (F.), Chimie minérale théorique et expérimentale, p. 186, Masson \& Cie, Paris, 1950.

[12] Rogers (K. C.), 7e C.I.P.I.G., Belgrade, 1965. 